\title{
SOIL MICROBIAL PROPERTIES IN COAL MINE TAILINGS UNDER REHABILITATION
}

\author{
S. CLAASSENS ${ }^{*}{ }^{1}-$ K.J. RIEDEL ${ }^{1}-$ L. VAN RENSBURG ${ }^{1}-$ T.L. MORGENTHAL ${ }^{2}-$ \\ P.J. JANSEN VAN RENSBURG ${ }^{1}$ \\ *e-mail:mkbsc@puk.ac.za \\ ${ }^{1}$ School of Environmental Sciences and Development, North-West University, \\ Potchefstroom Campus, Private Bag X6001, Potchefstroom, 2520, South Africa \\ (phone: +27 18 2992321, fax: +27 18 2992330) \\ ${ }^{2}$ GeoInformatics Division: ARC-ISCW, \\ Private Bag X79, 600 Belvedere Street, Pretoria, 0001, South Africa \\ (phone: +27 123102582 , fax +27 123231157 ) \\ *Corresponding author \\ (Received $5^{\text {th }}$ May 2005 , accepted $4^{\text {th }}$ August 2005)
}

\begin{abstract}
Microbial properties have been reported to be useful indicators of soil quality and could possibly serve as assessment criteria of successful rehabilitation of ecologically disturbed areas. During this study, the application value of microbial enumeration techniques and enzymatic assays as a measure of the progress of rehabilitation of coal discard sites was evaluated. Each site was analysed for physical and chemical characteristics of the topsoil; vegetation cover; potential enzymatic activities (dehydrogenase, B-glucosidase, urease, acid and alkaline phosphatase) and quantification of aerobic oligotrophic and copiotrophic bacteria and fungi (selective enumeration). The relationship between soil properties, vegetation cover and microbial properties was analysed using multivariate ordination techniques. Although the discard sites had different rehabilitation ages ( $1-8$ years), no statistically significant difference existed between these sites based on physical and chemical characteristics or selective enumeration $(p>0.05)$. Differentiation was possible based on enzymatic activities, where sites with relatively higher vegetation cover and organic carbon content had a positive association with enzymatic activities. Organic carbon content correlated significantly with $\beta$-glucosidase $(r=0.80$, $p<0.05)$, urease $(r=0.96, p<0.05)$, acid phosphatase $(r=0.76, p<0.05)$, dehydrogenase $(r=0.69, p<0.10)$ and microbial biomass $(r=0.73, p<0.10)$. The characterisation of microbial activity holds potential as complementary criteria for evaluating rehabilitation progress on mine discard sites.
\end{abstract}

Keywords: coal discard;enzymatic activity; rehabilitation; soil quality

\section{Introduction}

The soil environment constitutes a very important natural resource that should not only be preserved, but where possible also improved [1]. South African legislation requires developers to ecologically rehabilitate damaged environments, which calls for careful planning and implementation of sound ecological principles. One of the more critical aspects of the rehabilitation process is the improvement of the tailings material to sustain plant growth by the creation of a suitable growth medium. A control measure frequently applied for the containment of discard material is the application of a topsoil cover, which facilitates the subsequent revegetation of the site. The establishment of permanent, self-sustainable vegetation on most mine waste sites in South Africa is, however, problematic.

Microbial activity is fundamental in the processes that make energy and nutrients available for recycling in the ecosystem and soil microorganisms play crucial roles in the biogeochemical cycling of carbon $(\mathrm{C})$, nitrogen $(\mathrm{N})$, and phosphorus $(\mathrm{P})[2,3]$. The 
presence and activity of enzymes is vital for all biochemical transformations in soil, thus the study of soil enzymatic activities provides insight into microbial dynamics and populations [4]. Studies have shown that enzymatic activities such as that of dehydrogenase, $\beta$-glucosidase, urease, and phosphatase show significant correlation with total organic carbon (TOC), total nitrogen (TN), water-filled pore space (WFPS), and heterotrophic bacterial and fungal biomass $[2,5]$.

Dehydrogenase activity was assayed as an estimation of overall microbial activity and it has been reported to generally be directly related to organic carbon content of soil $[2,5]$. $\beta$-Glucosidase activity is related to the carbon cycle and is very useful in monitoring of soil quality due to its central role in the cycling of organic matter [6]. Urease and phosphatase are often measured because of their importance in the nitrogen and phosphorus cycles, respectively [5]. Enzymatic activities in relation to the cycling of nitrogen (ammonification, nitrification, denitrification) or phosphorus (release of inorganic phosphorus) in soil have been used to evaluate the fertility of the soil $[5,7]$.

This study was undertaken to determine whether microbial enumeration techniques and enzymatic assays could differentiate between coal discard sites of varying rehabilitation ages.

\section{Materials and methods}

\section{Site details}

The study was conducted on seven vegetated coal discard sites under rehabilitation and managed by Ingwe Mine Closure Operations, Ingwe Mines, South Africa (site identities are presented in Table 2.). The soil used as cover was excavated from adjacent borrow pits or stripped from the sites before mining. All the coal discard sites were vegetated with a grass seed mixture mostly dominated by the commercially available grasses Eragrostis tef, Eragrostis curvula, Chloris gayana, Digitaria eriantha, Cynodon dactylon, and Pennisetum clandestinum. As a management practice, all sites are defoliated at the end of each growing season. All sites were also treated with variable amounts of lime, inorganic fertilisers, and well-cured manure at the onset of rehabilitation and during annual maintenance according to lime requirement, fertiliser recommendation, and organic carbon content. The average application rates per site over a four-year period are indicated in Table 1. The addition of micro- and macroelements was performed according to the results obtained from a 1:2 water-extract soil analysis. Macro-elements ( $\mathrm{Ca}, \mathrm{Mg}$ and $\mathrm{K}$ ) were always added as nitrates and phosphorus was supplemented using super-phosphate. No soil profiles similar to that used as cover layer on the discard sites were available to use as control sites. Therefore, random sites from the surrounding areas were chosen as reference sites (Table 2). These sites were located in areas adjacent to three of the coal discard sites, were less impacted than the rehabilitation sites and representative of relatively natural veldt. All areas were located within the grassland biome of South Africa and receive an average annual rainfall between $700-800 \mathrm{~mm}$. 
Table 1. Average application rates $\left(\mathrm{kg} . \mathrm{ha}^{-1}\right)$ of fertiliser amendments per site over a four year period.

\begin{tabular}{|l|l|}
\hline Amendment & Application rate (kg. ha \\
\hline $\mathrm{CaNO}_{3}$ & $121.00 \pm 17.62$ \\
\hline Table 1. continued from page 2. \\
\hline Amendment & Application rate (kg. ha \\
\hline $\mathrm{MgNO}_{3}$ & $55.90 \pm 7.48$ \\
\hline $\mathrm{KNO}_{3}$ & $89.10 \pm 21.27$ \\
\hline Super-phosphate & $183.75 \pm 29.79$ \\
\hline Manure & $6361.11 \pm 2362.71$ \\
\hline Lime & $6800.00 \pm 3117.69$ \\
\hline \multicolumn{2}{|l}{ Values given are mean \pm standard error. } \\
\hline
\end{tabular}

\section{Sampling procedure}

A random sampling design was used to obtain three composite samples per site ( 5 cores per composite sample) of the soil cover from seven coal discard sites $(n=21)$ $[8,9]$ and three reference sites $(n=9)$. All samples were obtained mid-summer (November) in the same quadrates used for the assessment of vegetation growth. A soil auger was used to obtain volume samples with a minimum of $1 \mathrm{~kg}$ of soil per sampling area. The top $0-10 \mathrm{~cm}$ of the soil cover layer was sampled since most of the discard sites had an effective soil cover depth of only $\leq 15 \mathrm{~cm}$. Samples were obtained using aseptic techniques as described by Dick et al. [9]. Soil samples were placed in tightly sealed plastic bags and kept at $4^{\circ} \mathrm{C}$ to keep them field moist and to preserve biological properties. Composite (consolidated) samples were mixed thoroughly to contain equal weights of individual samples. Soil moisture content was determined gravimetrically after drying soil samples at $105^{\circ} \mathrm{C}$. Each consolidated sample was analysed within five days of sampling. The chemical and physical analyses for the characterisation of soil cover layers of the respective sites were done as previously described [10].

\section{Estimation of vegetation cover}

The ground and crown vegetation cover of all the sites were estimated in three $1 \mathrm{~m}^{2}$ quadrates randomly placed over a $50 \mathrm{~m}$ transect. The ground cover included all living and non-living organic material on the ground surface per area and the crown cover was regarded as the canopy cover spread of all grass species over a fixed area. Both values are expressed as a percentage per $\mathrm{m}^{2}$ surface area [11].

\section{Microbial counts}

Viable bacterial and fungal populations were determined by plating the dilution series onto selective media. Total heterotrophic bacteria were enumerated using cycloheximide agar, R2A, and soil extract agar (SEA). Sodium pyrophosphate $\left(\mathrm{Na}_{2} \mathrm{PO}_{4} \cdot 7 \mathrm{H}_{2} \mathrm{O} ; \mathrm{pH} 7.0\right)(0.1 \% \mathrm{~m} / \mathrm{v})$ solution was used as diluent for the bacteria [12]. Soil extract agar and R2A are selective media for oligotrophic and copiotrophic heterotrophic bacteria, respectively [13]. Soil extract agar was prepared specifically for each of the seven sites using soil from the relevant site. Fungi were enumerated by plating the dilution series onto rose bengal-streptomycin (RBS) agar [12]. Dextrin $(0.2 \% \mathrm{~m} / \mathrm{v})$ and dextrose $(0.2 \% \mathrm{~m} / \mathrm{v})$ solutions were used as diluents for suspension and dilution of the soil, respectively [8]. All inoculated media were incubated at room temperature $\left(22 \pm 3^{\circ} \mathrm{C}\right)$ for ca. 4-10 days. All microbial enumerations were carried out in duplicate. Data are reported as CFU g ${ }^{-1}$ dry soil. 
Table 2. Physical and chemical properties of the topsoil covers obtained from the seven coal discard sites and three reference sites.

\begin{tabular}{|c|c|c|c|c|c|c|c|c|c|c|}
\hline \multirow[t]{2}{*}{ Properties } & \multicolumn{7}{|c|}{ Rehabilitation sites } & \multicolumn{3}{|c|}{ Reference sites } \\
\hline & Newcastle & Ermelo & Hendrina & Bethal & Witbank 1 & Witbank 2 & Ogies & Newcastle & Ermelo & Ogies \\
\hline $\begin{array}{l}\text { Grid } \\
\text { Reference }\end{array}$ & $\begin{array}{l}27^{\circ} 51 ' 14^{\prime \prime} \mathrm{S} \\
29^{\circ} 57^{\prime} 23^{\prime \prime} \mathrm{E}\end{array}$ & $\begin{array}{l}26^{\circ} 29^{\prime} 41^{\prime \prime} \mathrm{S} \\
29^{\circ} 45^{\prime} 34^{\prime \prime} \mathrm{E}\end{array}$ & $\begin{array}{l}26^{\circ} 20^{\prime} 4^{\prime \prime} \mathrm{S} \\
29^{\circ} 53^{\prime} 19^{\prime \prime} \mathrm{E}\end{array}$ & $\begin{array}{l}26^{\circ} 10^{\prime} 8^{\prime \prime} \mathrm{S} \\
29^{\circ} 21^{\prime} 2, \mathrm{E}\end{array}$ & $\begin{array}{l}25^{\circ} 53^{\prime} 42^{\prime \prime} \mathrm{S} \\
29^{\circ} 10^{\prime} 27^{\prime} \prime \mathrm{E}\end{array}$ & $\begin{array}{l}25^{\circ} 54^{\prime} 35^{\prime \prime} \mathrm{S} \\
29^{\circ} 11^{\prime} 21^{\prime \prime} \mathrm{E}\end{array}$ & $\begin{array}{l}26^{\circ} 01 ' 22^{\prime \prime} \mathrm{S} \\
29^{\circ} 06^{\prime} 06^{\prime \prime} \mathrm{E}\end{array}$ & $\begin{array}{l}27^{\circ} 50^{\prime} \mathrm{S} \\
29^{\circ} 56^{\prime} \mathrm{E} \\
\end{array}$ & $\begin{array}{l}26^{\circ} 19^{\prime} \mathrm{S} \\
29^{\circ} 35^{\prime} \mathrm{E} \\
\end{array}$ & $\begin{array}{l}26^{\circ} 00^{\prime} \mathrm{S} \\
29^{\circ} 04^{\prime} \mathrm{E} \\
\end{array}$ \\
\hline $\begin{array}{l}\text { Rehabilitation } \\
\text { age (years) }\end{array}$ & 8 & 3 & 4 & 1 & 4 & 4 & 4 & N/A & N/A & N/A \\
\hline \multicolumn{11}{|l|}{$\begin{array}{l}\text { Chemical } \\
\text { Properties }\end{array}$} \\
\hline $\mathbf{C a}\left(\mathrm{mg} \mathrm{kg}^{-1}\right)$ & $\begin{array}{c}49.30 \pm \\
13.04 \\
\end{array}$ & $7.36 \pm 0.27$ & $3.73 \pm 1.68$ & $\begin{array}{l}25.10 \pm \\
9.72 \\
\end{array}$ & $2.92 \pm 0.53$ & $3.13 \pm 0.27$ & $5.65 \pm 1.29$ & $\begin{array}{c}0.05 \pm \\
0.02 \\
\end{array}$ & $\begin{array}{c}0.22 \pm \\
0.17 \\
\end{array}$ & $\begin{array}{c}0.10 \pm \\
0.02\end{array}$ \\
\hline $\mathbf{M g}\left(\mathrm{mg} \mathrm{kg}^{-1}\right)$ & $15.77 \pm 3.40$ & $2.08 \pm 0.22$ & $1.47 \pm 0.64$ & $7.89 \pm 4.41$ & $0.92 \pm 0.18$ & $0.98 \pm 0.16$ & $1.22 \pm 0.49$ & $\begin{array}{c}0.19 \pm \\
0.02 \\
\end{array}$ & $\begin{array}{c}0.33 \pm \\
0.15 \\
\end{array}$ & $\begin{array}{c}0.15 \pm \\
0.01 \\
\end{array}$ \\
\hline $\mathbf{K}\left(\mathrm{mg} \mathrm{kg}^{-1}\right)$ & $6.29 \pm 1.71$ & $5.31 \pm 0.78$ & $25.08 \pm 8.32$ & $5.90 \pm 1.96$ & $4.92 \pm 1.26$ & $12.59 \pm 6.51$ & $24.59 \pm 9.31$ & $\begin{array}{c}0.21 \pm \\
0.01 \\
\end{array}$ & $\begin{array}{c}0.18 \pm \\
0.07 \\
\end{array}$ & $\begin{array}{c}0.55 \pm \\
0.14 \\
\end{array}$ \\
\hline $\mathbf{N a}\left(\mathrm{mg} \mathrm{kg}^{-1}\right)$ & $2.08 \pm 0.30$ & $1.85 \pm 0.12$ & $1.21 \pm 0.44$ & $1.56 \pm 0.44$ & $0.93 \pm 0.12$ & $0.87 \pm 0.27$ & $1.21 \pm 0.17$ & $\begin{array}{c}0.13 \pm \\
0.07 \\
\end{array}$ & $\begin{array}{c}0.05 \pm \\
0.01 \\
\end{array}$ & $\begin{array}{c}0.03 \pm \\
0.00\end{array}$ \\
\hline $\mathrm{SO}_{4}\left(\mathrm{mg} \mathrm{kg}^{-1}\right)$ & $\begin{array}{c}239.00 \pm \\
72.57 \\
\end{array}$ & $28.27 \pm 2.17$ & $13.77 \pm 4.72$ & $\begin{array}{c}122.04 \pm \\
71.69 \\
\end{array}$ & $\begin{array}{c}25.62 \pm \\
12.21 \\
\end{array}$ & $26.34 \pm 6.06$ & $\begin{array}{c}32.62 \pm \\
15.90 \\
\end{array}$ & $\begin{array}{c}0.16 \pm \\
0.03 \\
\end{array}$ & $\begin{array}{c}0.18 \pm \\
0.06 \\
\end{array}$ & $\begin{array}{c}0.19 \pm \\
0.04 \\
\end{array}$ \\
\hline $\mathbf{N O}_{3}\left(\mathrm{mg} \mathrm{kg}^{-1}\right)$ & $2.45 \pm 1.23$ & $6.38 \pm 1.30$ & $16.19 \pm 6.59$ & $6.38 \pm 5.15$ & $1.31 \pm 0.16$ & $6.05 \pm 3.50$ & $11.45 \pm 7.31$ & $\begin{array}{c}0.08 \pm \\
0.04 \\
\end{array}$ & $\begin{array}{c}0.24 \pm \\
0.18 \\
\end{array}$ & $\begin{array}{c}0.25 \pm \\
0.03\end{array}$ \\
\hline $\mathbf{N H}_{4}\left(\mathrm{mg} \mathrm{kg}^{-1}\right)$ & $0.39 \pm 0.07$ & $0.26 \pm 0.07$ & $0.73 \pm 0.23$ & $0.30 \pm 0.04$ & $0.43 \pm 0.09$ & $0.64 \pm 0.07$ & $0.47 \pm 0.21$ & $\begin{array}{c}0.06 \pm \\
0.01 \\
\end{array}$ & $\begin{array}{c}0.03 \pm \\
0.01 \\
\end{array}$ & $\begin{array}{l}0.05 \pm \\
0.00\end{array}$ \\
\hline $\mathbf{C l}\left(\mathrm{mg} \mathrm{kg}^{-1}\right)$ & $2.59 \pm 0.18$ & $12.75 \pm 5.20$ & $5.71 \pm 1.96$ & $5.35 \pm 0.71$ & $1.69 \pm 0.32$ & $4.01 \pm 1.35$ & $3.03 \pm 1.03$ & $\begin{array}{c}0.30 \pm \\
0.09\end{array}$ & $\begin{array}{c}0.18 \pm \\
0.08 \\
\end{array}$ & $\begin{array}{c}0.33 \pm \\
0.11 \\
\end{array}$ \\
\hline $\mathbf{F e}\left(\mathrm{mg} \mathrm{kg}^{-1}\right)$ & $\begin{array}{c}33.44 \pm \\
18.89 \\
\end{array}$ & $\begin{array}{c}81.48 \pm \\
16.06 \\
\end{array}$ & $\begin{array}{c}5659.82 \pm \\
2273.28 \\
\end{array}$ & $\begin{array}{c}105.93 \pm \\
89.03 \\
\end{array}$ & $\begin{array}{c}5937.56 \pm \\
2048.50 \\
\end{array}$ & $\begin{array}{c}11956.04 \pm \\
743.35 \\
\end{array}$ & $\begin{array}{c}2126.28 \pm \\
878.29 \\
\end{array}$ & $\begin{array}{c}23.12 \pm \\
1.64 \\
\end{array}$ & $\begin{array}{c}21.52 \pm \\
3.61 \\
\end{array}$ & $\begin{array}{c}43.05 \pm \\
13.26 \\
\end{array}$ \\
\hline $\mathbf{M n}\left(\mathrm{mg} \mathrm{kg}^{-1}\right)$ & $\begin{array}{c}837.25 \pm \\
480.20 \\
\end{array}$ & $\begin{array}{c}16.45 \pm \\
15.02 \\
\end{array}$ & $\begin{array}{c}47.96 \pm \\
14.59 \\
\end{array}$ & $5.25 \pm 5.05$ & $19.90 \pm 5.57$ & $75.32 \pm 6.21$ & $29.99 \pm 6.24$ & $\begin{array}{c}2.56 \pm \\
0.42 \\
\end{array}$ & $\begin{array}{c}2.20 \pm \\
0.90 \\
\end{array}$ & $\begin{array}{c}3.49 \pm \\
0.63 \\
\end{array}$ \\
\hline $\mathbf{C u}\left(\mathrm{mg} \mathrm{kg}^{-1}\right)$ & $0.96 \pm 0.96$ & $3.99 \pm 3.99$ & $31.29 \pm 4.05$ & $6.86 \pm 1.94$ & $8.14 \pm 8.14$ & $19.63 \pm 8.16$ & $8.62 \pm 5.44$ & $\begin{array}{c}0.37 \pm \\
0.22 \\
\end{array}$ & $\begin{array}{c}0.88 \pm \\
0.42 \\
\end{array}$ & $\begin{array}{c}1.25 \pm \\
0.30 \\
\end{array}$ \\
\hline $\mathbf{Z n}\left(\mathrm{mg} \mathrm{kg}^{-1}\right)$ & $16.94 \pm 9.69$ & $6.74 \pm 6.50$ & $19.08 \pm 4.42$ & $5.59 \pm 2.77$ & $4.28 \pm 3.79$ & $21.88 \pm 6.96$ & $17.27 \pm 0.57$ & $\begin{array}{c}0.95 \pm \\
0.14 \\
\end{array}$ & $\begin{array}{c}1.36 \pm \\
0.76 \\
\end{array}$ & $\begin{array}{c}1.31 \pm \\
0.12 \\
\end{array}$ \\
\hline $\mathbf{B}\left(\mathrm{mg} \mathrm{kg}^{-1}\right)$ & $\begin{array}{c}35.36 \pm \\
35.36 \\
\end{array}$ & $2.72 \pm 2.72$ & $0.00 \pm 0.00$ & $\begin{array}{c}32.64 \pm \\
16.32 \\
\end{array}$ & $\begin{array}{c}130.54 \pm \\
64.76 \\
\end{array}$ & $\begin{array}{c}89.75 \pm \\
63.38 \\
\end{array}$ & $\begin{array}{c}127.83 \pm \\
67.34 \\
\end{array}$ & $\begin{array}{c}30.83 \pm \\
7.24 \\
\end{array}$ & $\begin{array}{c}22.50 \pm \\
10.52 \\
\end{array}$ & $\begin{array}{c}35.79 \pm \\
2.28 \\
\end{array}$ \\
\hline $\mathbf{P}\left(\mathrm{mg} \mathrm{kg}^{-1}\right)$ & $18.97 \pm 7.53$ & $5.60 \pm 0.84$ & $\begin{array}{c}146.43 \pm \\
65.07 \\
\end{array}$ & $\begin{array}{c}18.23 \pm \\
9.75 \\
\end{array}$ & $27.37 \pm 7.27$ & $\begin{array}{c}38.13 \pm \\
16.77 \\
\end{array}$ & $\begin{array}{c}96.77 \pm \\
17.27 \\
\end{array}$ & $\begin{array}{c}7.01 \pm \\
1.70 \\
\end{array}$ & $\begin{array}{c}5.51 \pm \\
0.69 \\
\end{array}$ & $\begin{array}{c}17.98 \pm \\
0.25\end{array}$ \\
\hline pH & $5.51 \pm 0.27$ & $6.20 \pm 0.26$ & $7.03 \pm 0.030$ & $7.41 \pm 0.44$ & $7.15 \pm 0.50$ & $6.24 \pm 0.41$ & $6.90 \pm 0.37$ & $\begin{array}{c}5.74 \pm \\
0.30 \\
\end{array}$ & $\begin{array}{c}6.66 \pm \\
0.48 \\
\end{array}$ & $\begin{array}{l}5.60 \pm \\
0.05 \\
\end{array}$ \\
\hline $\begin{array}{l}\text { Electrical } \\
\text { Conductivity } \\
\text { (EC) }\left(\mathrm{mS} \mathrm{m}^{-1}\right)\end{array}$ & $\begin{array}{l}117.33 \pm \\
46.25\end{array}$ & $21.67 \pm 4.10$ & $29.00 \pm 6.27$ & $\begin{array}{c}37.00 \pm \\
4.36\end{array}$ & $26.33 \pm 2.03$ & $18.67 \pm 6.23$ & $35.00 \pm 8.02$ & $\begin{array}{c}23.00 \pm \\
3.27\end{array}$ & $\begin{array}{c}29.75 \pm \\
10.90\end{array}$ & $\begin{array}{c}24.00 \pm \\
3.61 \\
\end{array}$ \\
\hline $\begin{array}{l}\text { \% Organic } \\
\text { Carbon }(\% \mathrm{C})\end{array}$ & $0.69 \pm 0.14$ & $0.52 \pm 0.10$ & $2.20 \pm 0.74$ & $0.31 \pm 0.16$ & $0.32 \pm 0.08$ & $0.67 \pm 0.01$ & $2.72 \pm 1.36$ & $\begin{array}{c}2.98 \pm \\
0.77 \\
\end{array}$ & $\begin{array}{c}2.44 \pm \\
0.75 \\
\end{array}$ & $\begin{array}{l}1.48 \pm \\
0.43 \\
\end{array}$ \\
\hline $\begin{array}{l}\text { Cation } \\
\text { Exchange } \\
\text { Capacity } \\
(\text { CEC) } \\
\text { cmol }(+) \cdot \mathrm{kg}^{-1} \\
\end{array}$ & $24.34 \pm 1.60$ & $17.55 \pm 1.92$ & $9.34 \pm 1.56$ & $7.06 \pm 1.77$ & $4.19 \pm 0.61$ & $4.90 \pm 1.01$ & $9.92 \pm 3.95$ & $\begin{array}{c}13.97 \pm \\
3.29\end{array}$ & $\begin{array}{c}22.35 \pm \\
8.39\end{array}$ & $\begin{array}{l}6.36 \pm \\
0.95\end{array}$ \\
\hline $\begin{array}{l}\text { S-value } \\
\text { cmol }(+) \cdot \mathrm{kg}^{-1}\end{array}$ & $13.12 \pm 0.17$ & $12.80 \pm 3.59$ & $9.58 \pm 2.81$ & $8.46 \pm 3.43$ & $4.18 \pm 1.07$ & $3.23 \pm 1.20$ & $\begin{array}{c}95.17 \pm \\
14.20 \\
\end{array}$ & $\begin{array}{l}8.76 \pm \\
2.68 \\
\end{array}$ & $\begin{array}{c}19.74 \pm \\
8.06 \\
\end{array}$ & $\begin{array}{c}3.50 \pm \\
0.91 \\
\end{array}$ \\
\hline $\begin{array}{l}\text { Base } \\
\text { Saturation } \\
\text { (Bsat) }(\%) \\
\end{array}$ & $54.33 \pm 3.07$ & $\begin{array}{c}70.13 \pm \\
12.70\end{array}$ & $\begin{array}{c}98.94 \pm \\
15.35\end{array}$ & $\begin{array}{c}112.29 \pm \\
17.54\end{array}$ & $\begin{array}{c}96.49 \pm \\
13.94\end{array}$ & $\begin{array}{c}61.28 \pm \\
13.34\end{array}$ & $6.90 \pm 0.37$ & $\begin{array}{c}56.43 \pm \\
6.78\end{array}$ & $\begin{array}{c}81.66 \pm \\
11.51\end{array}$ & $\begin{array}{c}53.34 \pm \\
5.67\end{array}$ \\
\hline $\begin{array}{l}\text { Lime } \\
\text { requirement } \\
(\text { Lreq })\left(\mathrm{t}_{\mathrm{h}} \mathrm{ha}^{-1}\right)\end{array}$ & $3.55 \pm 2.21$ & $8.05 \pm 0.19$ & $0.00 \pm 0.00$ & $0.00 \pm 0.00$ & $0.62 \pm 0.62$ & $1.62 \pm 1.04$ & $0.67 \pm 0.67$ & $\begin{array}{l}0.00 \pm \\
0.00\end{array}$ & $\begin{array}{c}0.00 \pm \\
0.00\end{array}$ & $\begin{array}{c}0.00 \pm \\
0.00\end{array}$ \\
\hline $\begin{array}{l}\text { \% Soil } \\
\text { Moisture }\end{array}$ & $6.61 \pm 0.35^{\mathrm{ac}}$ & $9.87 \pm 2.13^{\mathrm{c}}$ & $6.84 \pm 0.57^{\mathrm{ac}}$ & $\begin{array}{l}4.35 \pm \\
0.27^{\mathrm{ab}}\end{array}$ & $1.68 \pm 0.04^{b}$ & $\begin{array}{l}2.59 \pm \\
0.29^{\mathrm{ab}} \\
\end{array}$ & $\begin{array}{l}3.02 \pm \\
0.75^{\mathrm{ab}}\end{array}$ & $\begin{array}{c}10.50 \pm \\
1.45^{\mathrm{a}} \\
\end{array}$ & $\begin{array}{c}9.26 \pm \\
2.11 \mathrm{a} \\
\end{array}$ & $\begin{array}{c}3.79 \pm \\
1.31 \mathrm{a} \\
\end{array}$ \\
\hline \multicolumn{11}{|l|}{$\begin{array}{l}\text { Physical } \\
\text { Properties }\end{array}$} \\
\hline Sand $(\%)$ & $79.22 \pm 5.53$ & $39.80 \pm 2.62$ & $68.18 \pm 2.53$ & $\begin{array}{c}58.70 \pm \\
1.00 \\
\end{array}$ & $61.23 \pm 1.62$ & $68.12 \pm 2.61$ & $67.46 \pm 0.91$ & $\begin{array}{c}55.57 \pm \\
4.17 \\
\end{array}$ & $\begin{array}{c}58.09 \pm \\
11.12 \\
\end{array}$ & $\begin{array}{c}80.73 \pm \\
3.68 \\
\end{array}$ \\
\hline Silt (\%) & $8.46 \pm 1.96$ & $42.66 \pm 1.76$ & $23.42 \pm 2.84$ & $\begin{array}{c}34.27 \pm \\
1.54 \\
\end{array}$ & $26.27 \pm 2.61$ & $20.93 \pm 2.92$ & $25.25 \pm 2.83$ & $\begin{array}{c}23.67 \pm \\
4.48 \\
\end{array}$ & $\begin{array}{c}14.04 \pm \\
4.09 \\
\end{array}$ & $\begin{array}{l}9.43 \pm \\
2.27 \\
\end{array}$ \\
\hline Clay (\%) & $12.31 \pm 3.81$ & $17.55 \pm 0.86$ & $8.39 \pm 0.51$ & $7.02 \pm 1.63$ & $12.51 \pm 1.10$ & $10.95 \pm 1.75$ & $7.29 \pm 2.26$ & $\begin{array}{c}20.76 \pm \\
1.29 \\
\end{array}$ & $\begin{array}{c}27.87 \pm \\
7.48 \\
\end{array}$ & $\begin{array}{c}9.84 \pm \\
1.54 \\
\end{array}$ \\
\hline \multicolumn{11}{|l|}{$\begin{array}{l}\text { Biological } \\
\text { Properties }\end{array}$} \\
\hline $\begin{array}{l}\% \text { Ground } \\
\text { Cover }\end{array}$ & $\begin{array}{c}77.42 \pm \\
17.71^{\mathrm{abc}} \\
\end{array}$ & $\begin{array}{l}63.21 \pm \\
23.72^{\mathrm{a}} \\
\end{array}$ & $\begin{array}{l}71.25 \pm \\
19.10^{\text {cd }}\end{array}$ & $\begin{array}{l}41.57 \pm \\
15.67^{\mathrm{ab}} \\
\end{array}$ & $\begin{array}{l}28.92 \pm \\
12.30^{\mathrm{ab}}\end{array}$ & $\begin{array}{c}86.75 \pm \\
16.59^{\mathrm{d}} \\
\end{array}$ & $\begin{array}{l}60.91 \pm \\
14.97^{\text {bcd }} \\
\end{array}$ & $\begin{array}{l}59.00 \pm \\
11.00^{\mathrm{x}} \\
\end{array}$ & $\begin{array}{l}59.00 \pm \\
12.59 \mathrm{x} \\
\end{array}$ & $\begin{array}{c}100.00 \\
\pm 0.00 \mathrm{x} \\
\end{array}$ \\
\hline $\begin{array}{l}\text { \% Crown } \\
\text { Cover }\end{array}$ & $\begin{array}{c}56.29 \pm \\
19.85^{\mathrm{b}} \\
\end{array}$ & $\begin{array}{l}38.75 \pm \\
12.36^{\mathrm{b}} \\
\end{array}$ & $\begin{array}{l}80.13 \pm \\
12.95^{\mathrm{b}} \\
\end{array}$ & $\begin{array}{l}45.71 \pm \\
15.97^{\mathrm{a}} \\
\end{array}$ & $\begin{array}{l}42.50 \pm \\
22.11^{\mathrm{a}} \\
\end{array}$ & $\begin{array}{l}84.83 \pm \\
14.27^{\mathrm{b}} \\
\end{array}$ & $\begin{array}{l}65.91 \pm \\
19.98^{\mathrm{ab}} \\
\end{array}$ & $\begin{array}{c}67.00 \pm \\
8.89^{\mathrm{xy}} \\
\end{array}$ & $\begin{array}{c}52.50 \pm \\
9.68 \mathrm{x} \\
\end{array}$ & $\begin{array}{r}100.00 \\
\pm 0.00 \mathrm{y} \\
\end{array}$ \\
\hline
\end{tabular}

${ }^{1}$ Values given are mean \pm standard error

${ }^{2}$ Sites with the same combination of superscript alphabetic letters indicate no significant differences among sites 


\section{Soil enzymatic activities}

Before analyses, consolidated soil samples were passed through a $2 \mathrm{~mm}$ sieve. For the determination of dehydrogenase activity, soil was kept field moist, while air-dried samples were used for determination of $\beta$-glucosidase (EC 3.2.1.21), urease (urea amidohydrolase, EC 3.5.1.5), and acid (orthophosphoric monoester phosphohydrolase, EC 3.1.3.2, $\mathrm{pH}$ 6.5) and alkaline (orthophosphoric monoester phosphohydrolase, EC 3.1.3.1, $\mathrm{pH}$ 11.0) phosphatase activities [8]. All analyses were carried out in triplicate.

Dehydrogenase and urease (urea amidohydrolase, EC 3.5.1.5) activities were assayed according to the procedure as described by Alef \& Nannipieri [8]. B-glucosidase (EC 3.2.1.21) as well as acid (orthophosphoric monoester phosphohydrolase, EC 3.1.3.2, pH 6.5) and alkaline (orthophosphoric monoester phosphohydrolase, EC 3.1.3.1, pH 11.0) phosphatase activities were all based on $p$-nitrophenol release after cleavage of a synthetic substrate ( $p$-nitrophenyl glucoside and $p$-nitrophenyl phosphate, respectively) $[8,9]$. Modified universal buffer $\mathrm{pH} 6.5$ and $\mathrm{pH} 11.0$ were used for acid and alkaline phosphomonoesterase, respectively.

\section{Statistical analysis}

All samples $(n=21 ; n=9)$ were analysed in triplicate. The relationship between soil physical and chemical characteristics, the microbial and the vegetation variables was investigated using Principal Components Analysis (PCA) and Redundancy Analysis (RDA) multivariate ordination techniques using Canoco (Canoco for Windows Version 4.5, GLW-CPRO ${ }^{\circ}$ ). Principal Components Analyses were conducted on the soil physical and chemical variables, as well as on the vegetation cover and microbial properties analysed to determine how these variables were interrelated. A Redundancy Analysis (RDA) was subsequently performed with the activities of the five enzymes assayed, the microbial counts obtained using conventional techniques and the percentage ground and crown cover as species dependent variables and the most significant soil properties as independent environmental factors. The most significant soil physical and chemical variables were selected through the forward selection procedure provided in Canoco, thereby ensuring that only the most pertinent environmental gradients were investigated.

\section{Results and Discussion}

Differences could be observed between the reference and discard sites based on enzymatic activities. However, comparison between the reference and rehabilitation sites was problematic due to considerable differences between them, such as topography and soil depth. Results from the physical and chemical characterisation of the soil samples as well as the percentage vegetation cover are summarised in Table 2. The results obtained from the chemical analyses, showed high standard error values for most of the elements, indicating the heterogeneous nature of the topsoil layer or possible mixing of topsoil with underlying discard material. A Principal Components Analysis (PCA) (not shown) indicated that the soil used as cover varied markedly between sites based on physical and chemical characteristics, although this variation was not statistically significant. The variation observed could have a significant impact on the structural diversity of the bacteria and fungi present as well as on the rehabilitation and establishment of a self-sustaining vegetation cover at these sites. 
grass species showed great variation in their growth form - some had a small basal (ground) cover but a large crown cover (Table 2). The dominance of tufted grasses with a higher crown cover and a lower basal cover at Bethal and Witbank 1, could be the reason for the low percentage ground vegetation cover $(\leq 41.57 \%)$ observed at these sites. Reduced aboveground plant diversity has been reported to decrease the microbial diversity in the soil ecosystem and to disturb its normal functioning. A degraded plant cover generally results in lower soil organic matter content. Our results correspond with observations by other authors $[14,15]$ that decreased availability of organic matter relates to lower microbial activity. Bethal was characterised by a lower percentage vegetation cover $(\leq 45.71 \%)$ and related to lower estimated viable biomass $(7110 \mathrm{pmol}$ g-1 dry weight) and enzymatic activity (Fig. 1) compared to the other sites.

Statistical analysis of the microbial counts indicated no significant difference $(p>0.05)$ between sites based on microbial enumeration using a variety of culture media (Table 3). However, microbial enumeration indicated a higher abundance of copiotrophic microorganisms in discard sites than in reference sites. It is hypothesised that the abundance of microorganisms in the different sites could be classified along an $\mathrm{r}-\mathrm{K}$ gradient. The r-strategists rely upon high reproductive rates for survival within a community and prevail in environments where nutrients are readily available (copiotrophic), whereas the K-strategists depend upon physiological adaptations to the environmental resources [16]. When resources become scarce, r-strategists experience rapid reduction, whereas K-strategists tend to be successful in resource-limited situations (oligotrophic) [17].

The average activities of the enzymes assayed are presented in Table 3. The results indicate a lack of relationship between the type of phosphatase and soil $\mathrm{pH}$. This suggests that a variation in $\mathrm{pH}(5.51-7.41)$ was not a critical factor governing the predominance of acid or alkaline phosphatase activity in these ecosystems. An RDA ordination diagram illustrating the association between the dominant environmental variables, microbial enzymatic activities, microbial counts, and percentage ground and crown cover is presented in Fig. 1. Eigenvalues for the first two axes were 0.531 and 0.256 , respectively. Total observed variance of the first two canonical axes was $78.7 \%$. The first canonical axis correlated strongly with percentage organic carbon $(\% \mathrm{C})(\mathrm{r} 2=$ $0.8815)$ and the second axis correlated with $\mathrm{NH} 4+(\mathrm{r} 2=0.7817)$. According to a Monte Carlo Permutation test conducted with 499 permutations the first canonical axis was not statistically significant $(\mathrm{p}=0.054)$. The overall effect of the chosen environmental variables on the microbial enzymatic activities, however was statistically significant $(\mathrm{p}=0.0060)$.

Dehydrogenase activity was assayed as an estimation of overall microbial activity due to its presence in all microorganisms [13]. $\beta$-Glucosidase activity is related to the carbon cycle, fulfils a central role in the cycling of organic matter, is the most abundant of the three enzymes involved in cellulose degradation, and is rarely substrate limited [6]. Urease and phosphatase are often measured because of their importance in the nitrogen and phosphorus cycles, respectively [5]. Enzymatic activities in relation to the cycling of nitrogen (ammonification, nitrification, denitrification) or phosphorus (release of inorganic phosphorus) in soil have been used to evaluate the fertility of the soil or to describe the functioning of the ecosystem $[5,7]$. 
Table 3. Microbial counts and enzymatic properties of topsoil covers obtained from the seven coal discard sites and three reference areas.

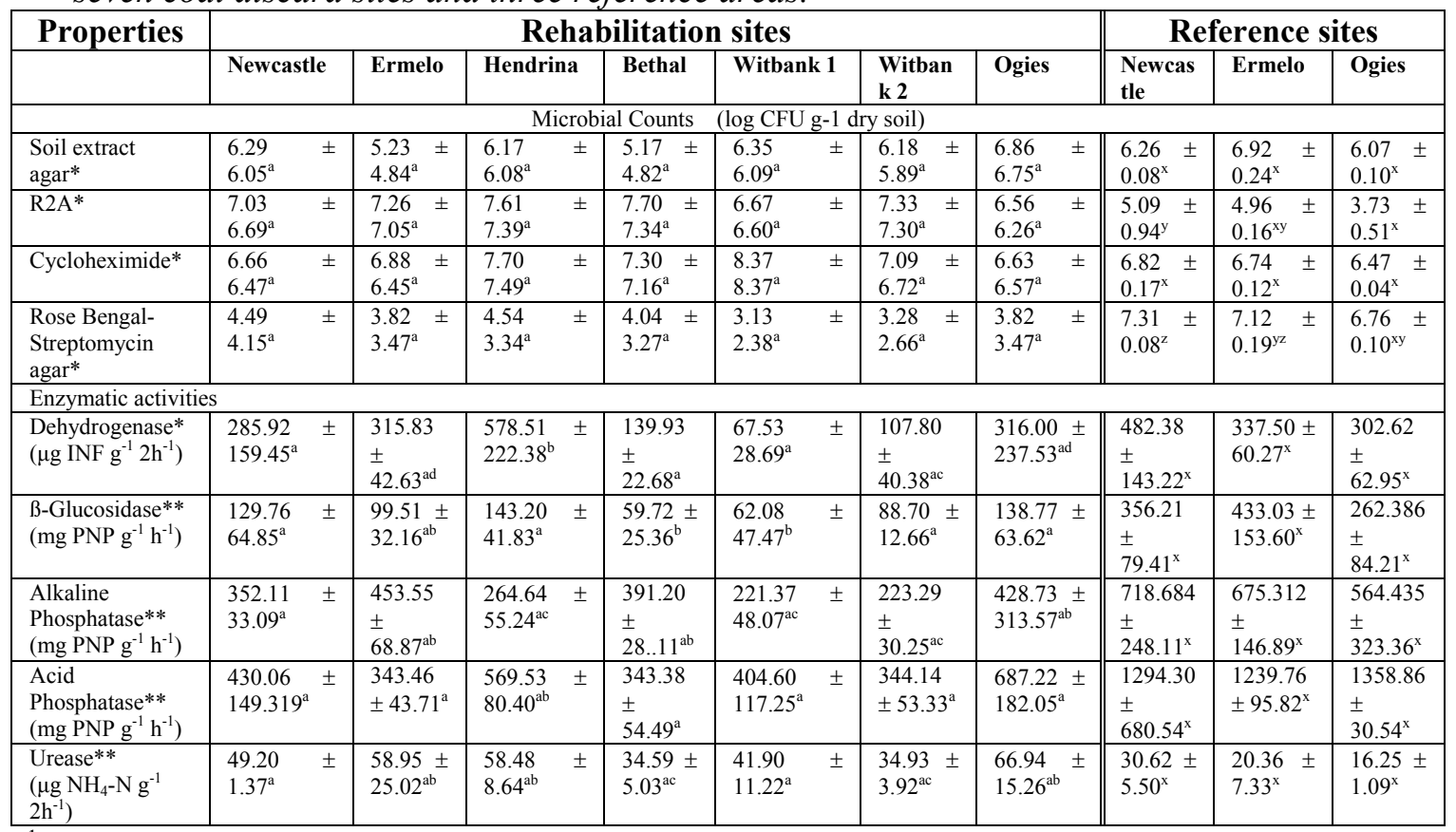

${ }^{1}$ All values \pm SEM represents the results obtained from three independent samples $(n=3)$ at a sampling depth of $0-15 \mathrm{~cm}$

$2 *$ Significant at the 0.05 probability level

$3 * *$ Significant at the 0.001 probability level

${ }^{4}$ Sites with the same combination of superscript alphabetic letters indicate no significant differences among sites

${ }^{5}$ INF: iodonitrotetrazolium chloride-formazan; PNP: para-nitrophenol

The Hendrina, Newcastle, and Ogies sites grouped to the right of the first ordination axis (Fig. 1) and were associated with higher overall enzymatic activity and vegetation cover. This may be attributed to the higher organic carbon content $(\geq 0.69 \%)$ (Table 2) present in these sites in comparison with the sites grouped to the left of the ordination diagram. The Bethal and Witbank 1 sites were characterised by lower organic carbon content $(\leq 0.32 \%)$ and associated lower ground cover $(\leq 41.57 \%)$. The RDA also showed these sites to have a negative/weak association with enzymatic activities. These results correspond with observations of Aon et al. [14] and Garcia et al. [15], which indicated a relationship between reduced aboveground plant diversity, lower soil organic matter, and decreased microbial diversity. The observation that organic carbon content had a significant correlation with $\beta$-glucosidase $(\mathrm{r}=0.80, p<0.05)$, acid phosphatase $(\mathrm{r}=0.96$, $p<0.05)$ and urease $(\mathrm{r}=0.76, p<0.05)$ (results not shown), emphasises the importance of organic matter as soil amendment during rehabilitation.

\section{Conclusions}

To obtain an accurate representation of the function and structure of soils, it is necessary to study the inter-relationship between physical, chemical, biochemical and biological properties. Measurement of only one or some of these properties will give only a partial evaluation of the state of the soil ecosystem. No single soil property is thus sufficient to evaluate the effect of anthropogenic or natural impacts on an ecosystem, because all methods are subject to limitations. It would therefore be most 
sensible to use a polyphasic approach - the combination of several types of techniques to assess soil quality.

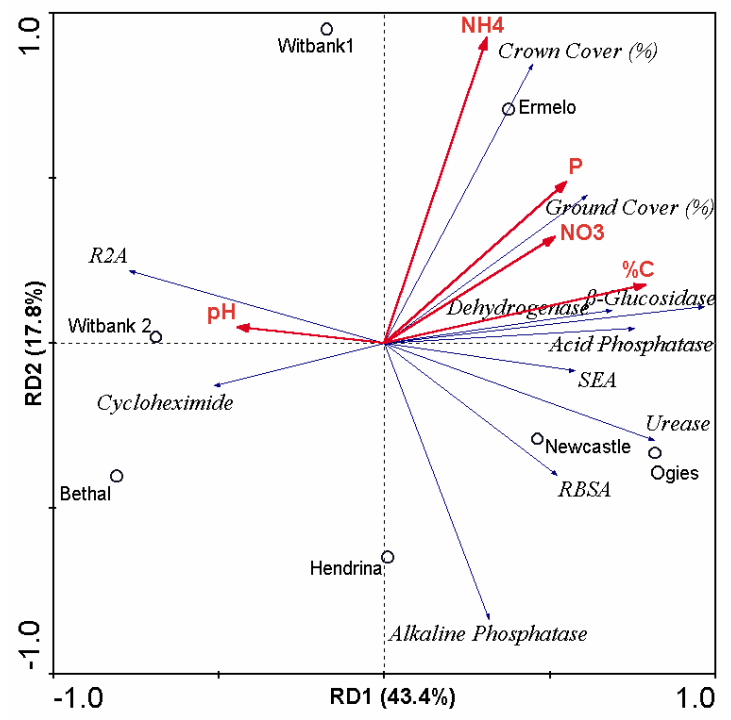

Figure 1. Redundancy analysis (RDA) ordination diagram illustrating the relationship between the dominant environmental variables, vegetation cover, and soil microbial and enzymatic properties of the seven discard sites. The environmental physical/chemical parameters are represented by bold vectors.

It is evident that the characterisation of microbial activity is sufficiently sensitive to differentiate between the soil covers of various coal discard sites and could be used as complementary assessment criteria to determine the rehabilitation status of sites associated with discard mining material. It also seems that a trend could exist between the progress of rehabilitation and $\mathrm{r}$ - and $\mathrm{K}$ - strategic microorganisms, with r-strategists favouring new sites or sites under fertiliser treatment and K-strategists favouring more stable environments. Clearly, a more detailed sampling study and in depth analysis, including spatial and temporal monitoring as well as appropriated DNA analysis is needed to investigate the observations made during this study.

Acknowledgements. This research was performed with the financial support of Ingwe Mine Closure Operations, Ingwe Mines, South Africa; and the National Research Foundation (NRF), South Africa.

\section{REFERENCES}

[1] Pascual, J.A., Garcia, C., Hernandez, T., Moreno, J.L., Ros, M. (2000): Soil microbial activity as a biomarker of degradation and remediation processes. - Soil Biology and Biochemistry 32: 1877-1883.

[2] Bandick, A.K., Dick, R.P. (1999): Field management effects on soil enzyme activities. - Soil Biology and Biochemistry 31: 1471-1479.

[3] Schoenholtz, S.H., Van Miegroet, H., Burger, J.A. (2000): A review of chemical and physical properties as indicators of forest soil quality: challenges and opportunities. - Forest Ecology and Management 138: 335-356. 
[4] Riffaldi, R., Saviozzi, A., Levi-Minzi, R., Cardelli, R. (2002): Biochemical properties of a Mediterranean soil as affected by long-term crop management systems. - Soil Tillage and Research 67: 109-114.

[5] Aon, M.A., Colaneri, A.C. (2001): Temporal and spatial evolution of enzymatic activities and physico-chemical properties in an agricultural soil. - Applied Soil Ecology 18: 255-270.

[6] Turner, B.L., Hopkins, D.W., Haygarth, P.M., Ostle, N. (2002): $\beta$-Glucosidase activity in pasture soils. - Applied Soil Ecology 20: 157-162.

[7] Brohon, B., Delolme, C., Gourdon, R. (2001): Complementarity of bioassays and microbial activity measurements for the evaluation of hydrocarbon-contaminated soils quality. - Soil Biology and Biochemistry 33: 883-891.

[8] Alef, K., Nannipieri, P. (Eds.) (1995): Methods in Applied Soil Microbiology and Biochemistry. New York: Academic Press. 576p.

[9] Dick, R.P., Breakwell, D.P., Turco, R.F. (1996): Soil enzyme activities and biodiversity measurements as integrative microbiological indicators. In: Doran, J.W., Jones, A.J. (Eds.), Methods for assessing soil quality, SSSA Special Publication 49, Soil Science Society of America, Madison, WI, pp 247-271.

[10] Van Rensburg, L., De Sousa Correia, R.I., Booysen, J., Ginster, M. (1998): Revegetation on a coal fine ash disposal site in South Africa. - Journal of Environmental Quality 27: 1479-1486.

[11] Van Rensburg, L., Maboeta, M.S., Morgenthal, T.L. (2004): Rehabilitation of codisposed diamond tailings: growth medium rectification procedures and indigenous grass establishment. - Water, Air, and Soil Pollution 154: 101-113.

[12] Frederickson, J.K., Balkwill, D.L. (1998): Sampling and Enumeration Techniques. In: Burlage, R.S., (Ed.), Techniques in microbial ecology, Oxford University Press, Inc., New York, pp 239-252.

[13] Taylor, J.P., Wilson, B., Mills, M.S., Burns, R.G. (2002): Comparison of microbial numbers and enzymatic activities in surface soils and subsoils using various techniques. - Soil Biology and Biochemistry 34: 387-401.

[14] Aon, M.A., Sarena, D.E., Burgos, D.E., Cortassa, S. (2001): (Micro)biological, chemical, and physical properties of soils subjected to conventional or no-till management: an assessment of their quality status. - Soil Tillage and Research 60: 173-186.

[15] Garcia, C., Hernandez, T., Roldan, A., Martin, A. (2002): Effect of plant cover decline on chemical and microbiological parameters under Mediterranean climate. - Soil Biology and Biochemistry 34: 635-642.

[16] Atlas, R.M., Bartha, R. (1998): Microbial ecology. $4^{\text {th }}$ Ed. Benjamin/Cummings Science Publishing, Menlo Park, California, 694p.

[17] Sarathchandra, S.U., Ghani, A., Yeates, G.W., Burch, G., Cox, N.R. (2001): Effect of nitrogen and phosphate fertilisers on microbial and nematode diversity in pasture soils. - Soil Biology and Biochemistry 33: 953-964. 